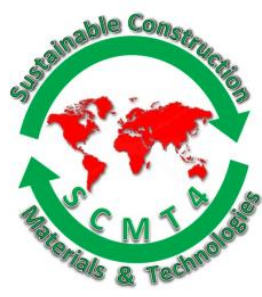

SCMT4

Las Vegas, USA, August 7-11, 2016

\title{
Long Term Durability Properties of Field Concretes with Glass Sand and Glass Powder
}

\author{
Tony Song ${ }^{1 a}$, Ion Dumitru ${ }^{1 b}$, and Bob Bornstein ${ }^{1 c}$ \\ ${ }^{1}$ Boral Construction Materials, Australia, P. O. Box 400, Winston Hills, NSW, Australia. \\ ${ }^{1 a}$ E-mail:<tony.song@boral.com.au >, ${ }^{1 b}$ Email :<ion.dumitru@boral.com.au>, \\ ${ }^{1 c}$ Email :<bob.bornstein@boral.com.au>.
}

\begin{abstract}
Efforts have been made to use the waste glass in concrete industry, either as the partial sand replacement, or as partial cementitious materials replacement. Two previous papers, regarding the intensive laboratory and field trials data, were presented in Ancona in 2010 and in Kyoto in 2013. Since glass itself is unstable in the alkaline environment of concrete, as a result, it is of great interest to assess the concrete durability properties and its performance after five years of trafficking. The durability properties have been tested in terms of alkaline silica reaction, if any, and interface zone with aggregate, water absorption, water sorptivity, water permeability, void contents, and chloride migration test. The performance assessment includes the compressive strength, the density and the wear resistance. This paper presents the feasibility and limitations when glass sand or glass powder is partially used in concrete mixes.
\end{abstract}

\section{INTRODUCTION}

The utilisation of waste glass in concrete industry has attracted a lot of interest worldwide due to the high disposal costs and environmental concerns. An intensive research work has been carried out since 2009 including two ways to use the waste glass in making concrete, partial sand replacement and partial cementitious materials replacement. The laboratory work was reported in Ancona, Italy [Dumitru et al. 2010]. Field trials and the field performance after one year service were presented in Kyoto, Japan [Dumitru et al. 2013]. Since the alkali silica reaction is the major concern when glass sand or glass powder is used in concrete manufacture, it is of the great importance to assess the relative longer term performance and durability properties of concrete with glass sand or with glass powder. This paper reports an on-going research work of the field concretes after five years in service.

\section{EXPERIMENTAL METHODOLOGY}

Three types of concretes were used to build a 100 meters long pavement at Boral's Dunmore Quarry, South of Sydney, Australia, including control concrete, concrete with glass sand and concrete with glass powder. The control concrete consists of compliant materials by local pavement specifications. For the glass sand concrete, the total sand in the control mix was replaced with $45 \%$ glass sand, while for the glass powder concrete, the total cementitious content in the control mix was replaced by $15 \%$ glass powder [Dumitru et al. 2010, 2013]. 
Visual inspection was carried out in order to get a general view of the surface wear after five years in service. For the pavement, the skid resistance is critical for its application. The Pendulum Friction Test has been performed on site as per AS 1141.42 over two spots for each concrete.

Twelve core samples from each concrete were extracted from the field pavement in June 2015 and tested for physical properties, durability performance, and alkali silica reaction.

Physical properties included the compressive strength and density. Compressive strength is the convenient method to assess how good the concrete became after five years in the field. The test methods used were AS 1012.14 and AS 1012.9, with the density being determined by water displacement AS 1012.12.2.

Durability properties included the voids content, sorptivity, water permeability, and chloride diffusion tests. The apparent volume of permeable voids content was determined by the boiled method as per AS1012.21. The sorptivity test by ASTM C1585 was to assess how quick the water was taken up due to the capillary effect. By contrast, the water permeability by DIN 1048 was to assess the water penetration depth due to the presence of pressure of $500 \mathrm{kpa}$ over a period of 72 hours. In addition, the chloride migration test is a widely acknowledged method to obtain the chloride diffusion coefficient, which can be further used to predict the service life. NT Build 492 was adopted for this purpose.

Alkali silica reaction is always questioned when the waste glass is used in the concrete. The laboratory has confirmed that this was not an issue during the mix design developing stage. [Dumitru et al. 2010]. However, a great interest is to assess the filed concrete. The concrete cores were examined by petrographic method as per ASTM C856 in order to fully understand the ASR issue, if any, after five years. In addition, the approximate composition of each concrete can be estimated so that the percentage of the perceived potential ASR component became known.

\section{SURFACE WEAR AND SKID FRICTION VALUE}

Visual inspection, after five years in service, has concluded that all concrete pavement segments are in a reasonable condition and surface paste has been obviously worn off due to the very heavy traffic over five years. The surface wear appears similar as shown in Figure 1.

The skid resistance is a major factor in road safety for concrete pavement work. For the pavement in service, the skid resistance performance is dependent on several factors including traffic condition, age of pavement, wet or dry surface, type of surface finishing, microstructure and macrostructure from concrete aggregates. Since these three trial concrete pavements were built in February 2010 and very close to each other, about 20 meters long of each section, the traffic condition, weather effect, finishing and coarse aggregate were similar. However, the application of glass sand or glass powder (probably as filler or "micro-aggregate") had the influence on the microstructure and macrostructure of the road concrete, consequently, the skid friction value would be different. For each pavement, two spots were randomly selected for the Pendulum test as per AS 1141.42. The friction values are presented in Table 1.

The marginal lower friction value for glass sand concrete and glass powder concrete is expected. However, the test friction value of 61 is much higher than the minimum required value of $44-48$ by the local road authority specification. This indicates that, in terms of skid resistance, it is safe when $45 \%$ glass sand is partially replaced the natural sand for glass sand concrete and also when $15 \%$ glass powder as the cementitious replacement for glass powder concrete. 

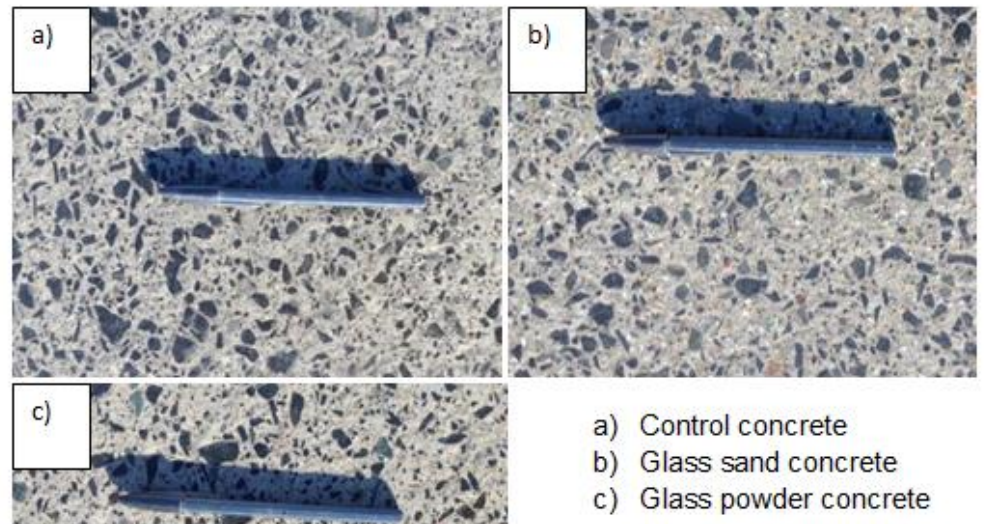

Figure 1. Surface Wear Appearance

Table 1. Pendulum friction value

\begin{tabular}{|c|c|c|c|c|}
\hline \multicolumn{2}{|c|}{ Concrete type } & Control & Glass sand & Glass powder \\
\hline Pendulum & Spot 1 & 65 & 61 & 62 \\
\cline { 2 - 5 } friction test AS & Spot 2 & 65 & 61 & 60 \\
\cline { 2 - 5 } 1141.42 & Average & $\mathbf{6 5}$ & $\mathbf{6 1}$ & $\mathbf{6 1}$ \\
\hline
\end{tabular}

\section{PHYSICAL PROPERTIES}

Two cores were tested for each concrete and results are presented in the Table 2.

Table 2. Compressive strength and density

\begin{tabular}{|c|c|c|c|c|c|}
\hline \multicolumn{3}{|c|}{ Concrete type } & Control & Glass sand & Glass powder \\
\hline \multirow{3}{*}{$\begin{array}{c}\text { Compressive strength AS } \\
1012.14 \text { and AS } 1012.9\end{array}$} & \multirow{3}{*}{$\mathrm{MPa}$} & Core 1 & 52.5 & 56.5 & 45.0 \\
\hline & & Core 2 & 54.5 & 56.0 & 44.0 \\
\hline & & Average & 53.5 & 56.3 & 44.5 \\
\hline \multirow{3}{*}{ Density AS 1012.12.2 } & \multirow{3}{*}{$\mathrm{Kg} / \mathrm{m}^{3}$} & Core 1 & 2360 & 2350 & 2280 \\
\hline & & Core 2 & 2340 & 2330 & 2280 \\
\hline & & Average & 2350 & 2340 & 2280 \\
\hline
\end{tabular}

It was noted that both the glass sand concrete and the control concrete have almost similar density and compressive strength. This well agrees with the findings by [Byars et al. 2004] that concrete with glass sand as an aggregate can have equivalent properties as concrete without glass sand. By contrast, the glass powder concrete has about $60 \mathrm{~kg} / \mathrm{m}^{3}$ lower density and about $9 \mathrm{MPa}$ lower compressive strength.

The lower compressive strength probably indicates that the glass powder used in this project would not be a type of pozzolan. Instead, it possibly works as a filler or "micro-aggregate", filling a grading gap 
between the fine sand and the cementitious component. It is important to note that the glass powder concrete has the core compressive strength still higher than the required minimum strength by local road authority specification which was targeted for this project.

The lower density of glass powder concrete is due to the lower density of glass powder itself. The glass powder has its density of 2.48 , quite lower in comparison with the combined cement $\&$ fly ash density of 3.03. When the glass powder was used at $60 \mathrm{~kg} / \mathrm{m}^{3}$ to partially replace cement and fly ash, this would result in about $33 \mathrm{~kg} / \mathrm{m}^{3}$ lower density than the control mix. In addition, the presence of more voids in the glass powder concrete must have contributed to the lower density as well.

\section{DURABILITY PROPERTIES}

Five tests have been performed to assess the durability properties. The results are presented in Table 3 and discussed as follows.

Table 3. Durability properties

\begin{tabular}{|c|c|c|c|c|c|c|}
\hline \multicolumn{4}{|c|}{ Concrete type } & Control & Glass sand & $\begin{array}{l}\text { Glass } \\
\text { powder }\end{array}$ \\
\hline \multirow{3}{*}{$\begin{array}{l}\text { Water absorption } \\
\text { initial rate }\end{array}$} & \multirow{3}{*}{$\begin{array}{l}\text { ASTM } \\
\text { C1585 }\end{array}$} & \multirow{3}{*}{$\underset{5}{\mathrm{~mm} / \mathrm{sec}^{0}}$} & Core A & 0.000734 & 0.000789 & 0.002186 \\
\hline & & & Core B & 0.001342 & 0.000892 & 0.002161 \\
\hline & & & Average & 0.001038 & 0.000841 & 0.002174 \\
\hline \multirow{3}{*}{$\begin{array}{l}\text { Water absorption } \\
\text { secondary rate }\end{array}$} & \multirow{3}{*}{$\begin{array}{l}\text { ASTM } \\
\text { C1585 }\end{array}$} & \multirow{3}{*}{$\underset{5}{\mathrm{~mm} / \mathrm{sec}^{0}}$} & Core A & 0.000503 & 0.000334 & 0.001152 \\
\hline & & & Core $\mathrm{B}$ & 0.000655 & 0.000499 & 0.001039 \\
\hline & & & Average & 0.000579 & 0.000417 & 0.001096 \\
\hline \multirow{3}{*}{$\begin{array}{c}\text { Water permeability } \\
\text { test }\end{array}$} & \multirow{3}{*}{$\begin{array}{l}\text { DIN } \\
1048\end{array}$} & \multirow{3}{*}{$\mathrm{mm}$} & Core I & 43.0 & 32.0 & 66.0 \\
\hline & & & Core II & 45.0 & 34.0 & 49.0 \\
\hline & & & Average & 44.0 & 33.0 & 57.5 \\
\hline \multirow{3}{*}{$\begin{array}{l}\text { Apparent volume } \\
\text { of permeable voids }\end{array}$} & \multirow{3}{*}{$\begin{array}{c}\text { AS } \\
1012.21\end{array}$} & \multirow{3}{*}{$\%$} & $\mathrm{Ai}$ & 5.7 & 6.0 & 6.8 \\
\hline & & & $\mathrm{Ab}$ & 5.9 & 6.3 & 7.1 \\
\hline & & & AVPV & 13.1 & 13.9 & 15.4 \\
\hline $\begin{array}{c}\text { Chloride Migration } \\
\text { test }\end{array}$ & NT 492 & $\mathrm{~m}^{2} / \mathrm{sec}$ & Average & 3.70E-12 & 2.88E-12 & 4.44E-12 \\
\hline
\end{tabular}

\section{Water absorption by ASTM C1585 method}

The performance of concrete subjected to many aggressive environments is a function of the penetrability of the pore system. In unsaturated concrete, the rate of ingress of water is largely controlled by absorption due to capillary rise [Hall 1989]. In this test method, only one surface is exposed to water at room temperature, while the other surfaces are sealed simulating water absorption in a member that is in contact with water on one side only.

As seen from Table 3, the glass sand concrete performed better than the control concrete, in terms of both the initial and secondary water absorption rates. By contrast, the glass powder concrete showed lower performance, probably due to more voids or/and microcracks. 


\section{Water permeability DIN 1048 test}

The permeability test gives a measure of the resistance of concrete against the penetration of water exerting pressure of 500kpa over a period of 3 days. The maximum depths of penetration obtained from the average of three specimens are presented in Table 3.

The glass sand concrete had a lower water penetration depth, of about $75 \%$, in comparison with the control concrete. The glass powder concrete showed a water penetration depth of about $31 \%$ higher than the control.

\section{Total apparent volume of permeable void content}

The parameter of permeable voids content has more than 20 years of history in practice in Australia and has been used in assessing the in-situ quality of concrete [Andrews-Phaedonos, 2012]. The total apparent volume of permeable voids is determined by the test method AS 1012.21 in which the specimens are oven dried, immersed completely in water at $23^{\circ} \mathrm{C}$, and then boiled under water for 6 hours. The results, as shown in Table 3, represent the maximum amount of water can be absorbed by a dry specimen.

The results show that the control concrete has marginally less voids content than the glass sand concrete. Both have the AVPV in the range of $13-14 \%$, less than the maximum value of 14 for the best grade of concrete by testing core samples (regardless of testing age) [VicRoads 2009]. By contrast, the concrete with glass powder has more voids, indicating the matrix is relatively porous. In terms of performance assessment [VicRoads 2009], concrete with glass power is one grade down than the control. This well agrees with previous data, such as the lower density (Table 2), higher water sorptivity (Table 3) and deeper water penetration depth by DIN 1048 test (Table 3).

\section{Chloride migration NT Build 492 test}

In Australia, NT Build 492 test is commonly used to test concrete mixes for use in chloride bearing environment. The results are presented in Table 3 .

It was noticed that the glass sand concrete is about $22 \%$ better than the control concrete. This result is in line with the data of water sorptivity and water permeability DIN 1048 tests of the field concrete cores in Table 3. This also agrees with the laboratory data when the mix design was developed [Dumitru et al. 2010]. In comparison, the glass powder concrete is about $20 \%$ worse than the control, which is consistent with other durability tests in Table 3, showing the poor durability performance of glass power concrete.

\section{PETROGRAPHIC EXAMINATION}

According to the document SA HB79:2015 [1], alkali aggregate reaction - guidelines on minimising the risk of damage to concrete structures in Australia, petrographic examination provides a quick screen for the potential of an aggregate alkali reaction if a concrete is affected by AAR by identifying reaction minerals and alkali reaction products as well. Therefore, cores taken from the field trialled area using glass as cementitious materials replacement and glass as fine aggregate replacement, together with a core for the "control" area (only natural aggregate) have been petrographic examined (ASTM C856) in order to assess the potential for AAR effect in the 5 years in-situ concrete.

Brief point counts were performed with the yielded results in volume percent being based on integration of the results of a count of 100 widely space points in thin section. 
The approximate composition of the concrete in the core is presented in Table 4. The components with perceived potential for alkali-silica reaction are shown in italics.

Table 4: Approximate composition of each concrete

\begin{tabular}{|l|c|c|c|}
\hline Concrete type & Control & Glass powder & Glass sand \\
\hline Coarse aggregate (Latite) & 39 & 36 & 43 \\
\hline Fine aggregate & 32 & 35 & 27 \\
\hline$-\quad$ quartz & 14 & 14 & 11 \\
\hline- Quartz- strained & 6 & 5 & 3 \\
\hline- Glass powder fragments & --- & 4 & --- \\
\hline- Glass fragments & --- & --- & 5 \\
\hline- Feldspar & 1 & 1 & $<1$ \\
\hline- Latite & 9 & 9 & 7 \\
\hline- Shell content & 1 & $<1$ & 1 \\
\hline- Free mineral grains & 1 & 2 & $<-$ \\
\hline- Acid volcanics & $<1$ & --- & 29 \\
\hline Hardened cement & 28 & 28 & 1 \\
\hline Air bubbles & 1 & 1 & \\
\hline
\end{tabular}

The petrographic examination, as showed in Figures 2, 3, 4, has noticed that:

(1) Overall all three concretes are essentially sound in petrographic examination;

(2) Both the coarse latite aggregate and fine aggregates (natural sand) are considered to be innocuous in relation to AAR and accordingly are interpreted to be suitable for use in concrete;

(3) For the control concrete, patchy porosity and very fine cracks are noted around aggregate fragments, sand grains. In addition, some air bubbles are present in the cement paste carrying needles of etttringite;

(4) For the glass sand concrete, small spherical to ovoid air bubbles are unevenly distributed, while porosity and cracks were noted around aggregate fragments, sand grain and intersecting air bubbles with the cement paste. It was noted that the air bubbles carry needles of etttringite;

(5) For glass powder concrete, small, spherical to ovoid porosity and cracks are noted around aggregate fragments, inserting air bubbles within the hardened paste.

Considering the petrographic examination carried out for control concrete core and the two cores with glass powder as cementitious materials replacement and glass sand as fine aggregate replacement it is concluded that:

There are no differences in concrete quality between the control mix and the two mixes using glass sand and glass powder;

- $\quad$ The concretes are sound;

- $\quad$ After five years in situ, there are no signs of AAR that may be caused by the glass sand or glass powder. 


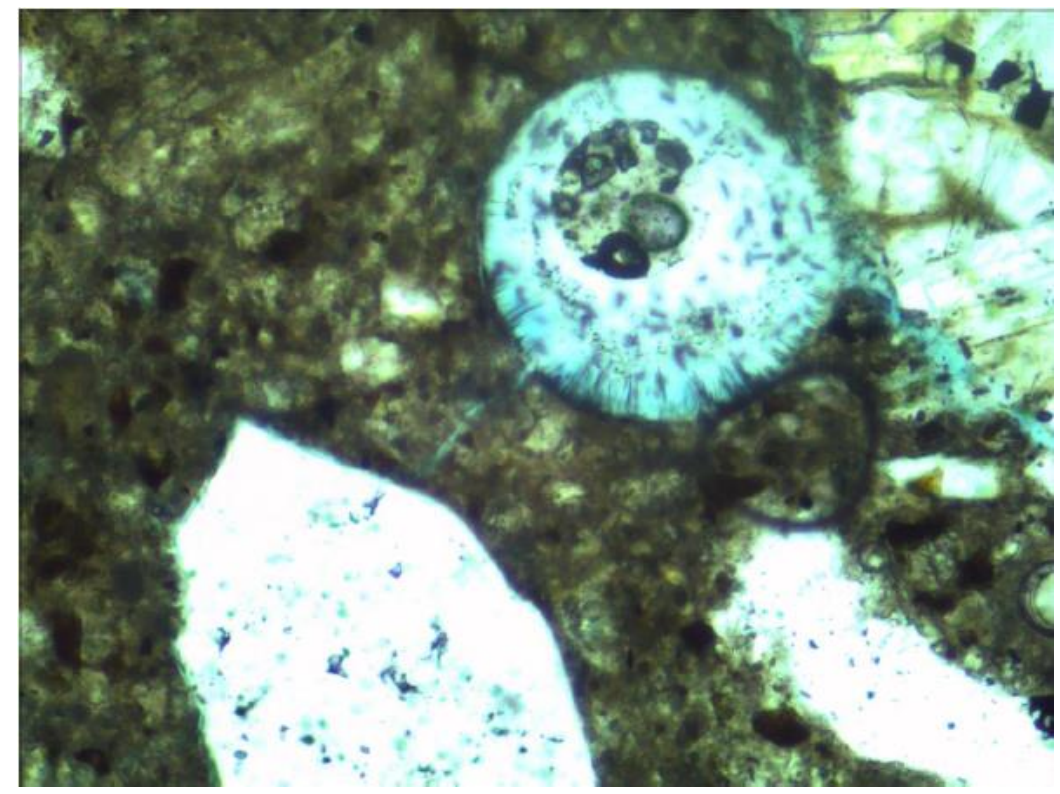

Figure 2. Petrographic Examination of Control Concrete

Medium-high magnification, plain transmitted light image of part of concrete displaying a coarse latite aggregate (upper right) showing a crack along its edge intersecting an air bubble, the crack then passing through cement matrix and running along around edges of a quartz gran. Note the radial needles of etttringite lining the air bubble. Field of view is $0.3 \mathrm{~mm}$.

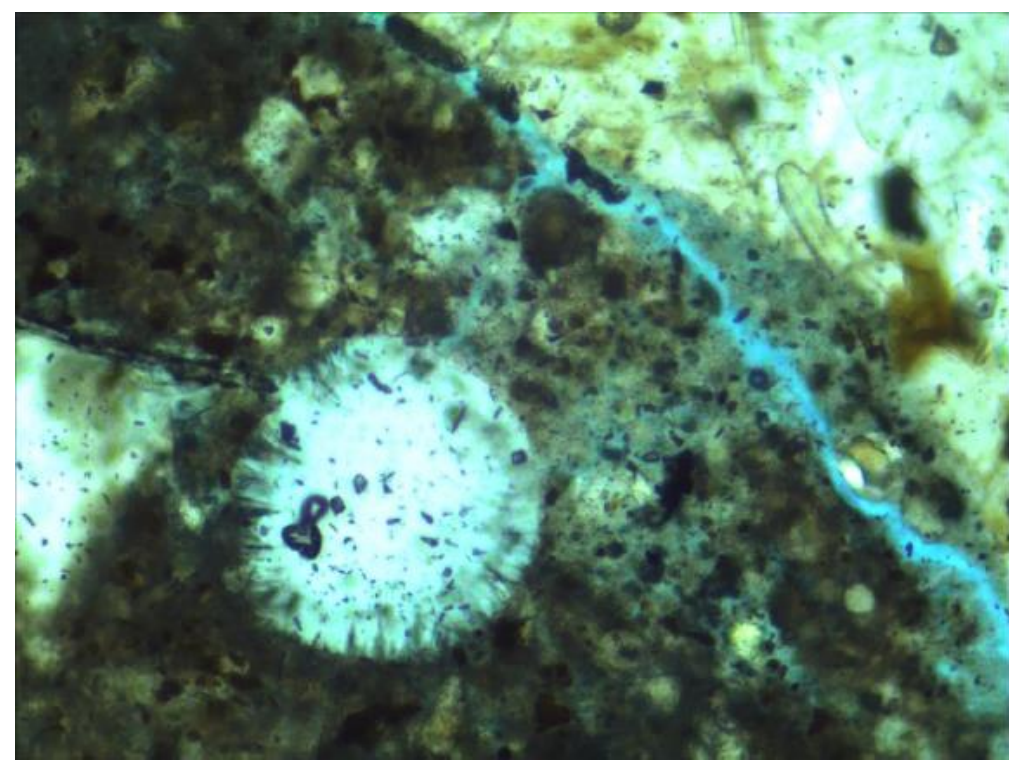

Figure 3. Petrographic Examination of Concrete with Glass Sand

Medium-high magnification, plain transmitted light image of part of concrete displaying a coarse latite aggregate (upper right) showing a crack along its edge. Note the radial needles of etttringite lining the air bubble and area of porosity between air bubble and latite aggregate. Field of view is $0.3 \mathrm{~mm}$. 


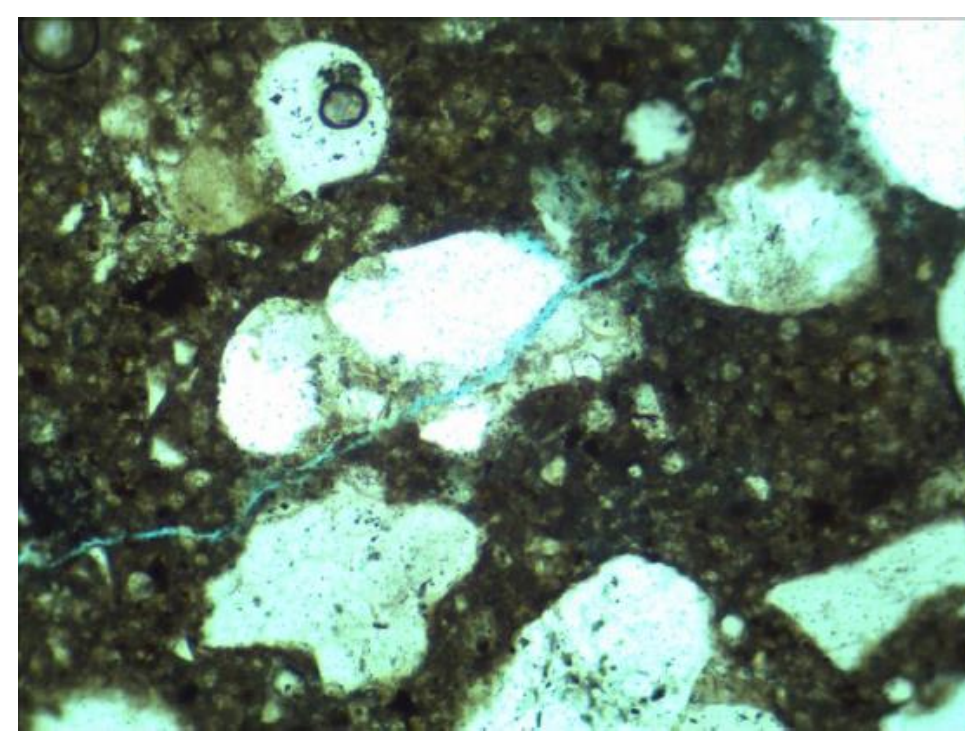

\section{Figure 4. Petrographic Examination of Concrete with Glass Powder}

Medium magnification, plain transmitted light image of part of concrete displaying an empty crack passing through a quartz sandstone clast. Field of view is $0.59 \mathrm{~mm}$.

\section{CONCLUSION}

The recycled glass sand can be used to partially (up to $45 \%$ in this study) replace natural sand in pavement concrete, achieving equivalent or better hardened properties, in terms of density, compressive strength, sorptivity, water permeability, chloride diffusion coefficient and the apparent volume of permeable voids.

The skid resistance of concrete with glass sand is 61 , about 4 units lower than the control concrete of 65 , but is still significantly higher than the minimum requirement of $44-48$ by the local road authorities.

The concretes with glass sand and glass powder are essential as sound as the control concrete using the natural sands. After five years in situ, there are no signs of alkali silica reaction that may be caused by the glass sand or glass powder.

Powdered glass can be used to partially replace cement. The replacement of $15 \%$ seems a bit high due to the lower compressive strength, more voids, higher sorptivity and high water permeability. However, we consider that more research in this area is needed.

\section{ACKNOWLEDGEMENT}

The authors gratefully acknowledge the support of Boral Materials Technical Services and approval to publish this paper. The opinions expressed in this paper are entirely those of the authors and not necessarily the policies and practices of the organisation they represent 


\section{REFERENCES}

Andrews-Phaedonos, F. (2012), "Assessment of Concrete durability using a single parameter with a high level of precision - the VPV test", $25^{\text {th }}$ ARRB Conference - Shaping the future: Linking policy, research and outcomes, Perth, Australia, pp1-15.

AS 1012.9 Australia Standard (1999), Methods of testing concrete method 9: Determination of the compressive strength of concrete specimens, 13 pages.

AS 1012.12.2 Australia Standard (1998), Methods of testing concrete Method 12.2: Determination of mass per unit volume of hardened concrete - water displacement method, 5 pages.

AS 1012.14 Australian Standard (1991), methods of testing concrete Method 14: Method for securing and testing cores from hardened concrete for compressive strength, 7 pages.

AS 1012.21 Australian Standard (1999), Method of testing concrete method 21: Determination of water absorption and apparent volume of permeable voids in hardened concrete, 9 pages.

AS 1141.42 Australian Standard (1999), Method for sampling and testing aggregates method 42: Pendulum friction test, 13 pages.

ASTM C856-14 Standard practice for petrographic examination of hardened concrete, 17 pages.

ASTM C1585 -11 Standard test method for measurement of rate of absorption of water by hydraulic cement concretes, 7 pages.

Byars, E., Zhu, H. and Morales, B. (2004), “ConGlassCrete 1 Project. Final Report”, United Kingdom.

Byars, E, Morales, B. and Zhu, H. (2004). "ConGlassCrete 2 Project. Final Report", United Kingdom.

DIN 1048 part 5 - Testing concrete, clause 7.6 water permeability.

Dumitru, I., Song, T., Caprar, V., Brooks, P., and Moss, J., (2010), "Incorporation of recycled glass for durable concrete", Second International Conference on Sustainable Construction Materials and Technologies, Universita Polytechnica delle Marche, Ancona, Italy, 9 pages.

Dumitru, I., Song, T., Bornstein, B., Brook, P. and Moss, J., (2013), "Field trials using recycled glass as natural sand replacement and powdered glass as cementitious materials replacement in concrete pavement", Third International Conference on Sustainable Construction Materials and Technologies, Kyoto, Japan, 9 pages.

Hall, C., "Water Sorptivity of Mortars and Concretes: A Review," Magazine of Concrete Research, Vol. 41, No. 147, June 1989, pp. 51-61.

NT build 443 - Accelerated chloride penetration test, 5 pages.

NT build 492 - Chloride migration coefficient from non-steady state migration experiments, 8 pages

VicRoads Standard Specification (2009), "Section 610 Structural Concrete", 45 pages. 